\title{
Three-Dimensional Virtual Anatomic Fit Study for an Implantable Pediatric Ventricular Assist Device
}

\author{
Arielle Drummond ${ }^{1}$, Timothy Bachman ${ }^{2}$, and James Antaki ${ }^{1}$ \\ ${ }^{1}$ Department of Biomedical Engineering, \\ Carnegie Mellon University, \\ 700 Technology Drive, Pittsburgh PA 15219 \\ \{adrummon, antaki\} @andrew. cmu.edu \\ ${ }^{2}$ Department of Bioengineering, University of Pittsburgh, \\ 749 Benedum Hall, Pittsburgh, PA 15213 \\ tbachmandengr.pitt.edu
}

\begin{abstract}
An innovative pediatric ventricular assist device (PVAD) is being developed to treat young patients $(2.5 \mathrm{~kg}-15 \mathrm{~kg})$ with severe heart failure that otherwise have very few options due to their small size. To optimize the design of the PVAD for the target patient population, three-dimensional anatomical compatibility studies must be conducted. The aim of this project was to evaluate the utility of three dimensional reconstructions to obviate fit studies in human subjects. Serial CT scans of the thorax of one child were obtained as part of routine treatment. The images were enhanced by adjusting the contrast of the images and segmented semi-automatically prior to 3-D reconstruction. The results were visualized as surface renderings of the rib cage and heart. This data was then amended with solid models of the implantable hardware, including the PVAD and cannulae. Manipulation of the relative orientation of the components revealed surgical challenges that may be anticipated and motivated design modifications to improve the anatomic compatibility. Unique challenges associated with these data sets include the availability of pediatric CT images and difficulty of segmentation due to the small scale of the anatomic features as compared to the resolution of the images.
\end{abstract}

\section{Introduction}

The limited options to treat ventricular failure in children with congenital heart disease has motivated the development of a highly reliable and biocompatible ventricular assist device (VAD) for chronic support (up to six months) for children $2.5 \mathrm{~kg}$ to $15 \mathrm{~kg}$. An innovative pediatric VAD (PVAD) is being developed by a consortium from the University of Pittsburgh, Carnegie Mellon University, World Heart Corporation (Oakland, CA) and LaunchPoint Technologies (Goleta, CA) based on a miniature axial flow pump with magnetic levitation. Design requirements to assure biocompatibility include minimization of blood trauma and anatomic compatibility of the implanted components, including the pump, cannulae sets, and drive line (See Figure 1). 

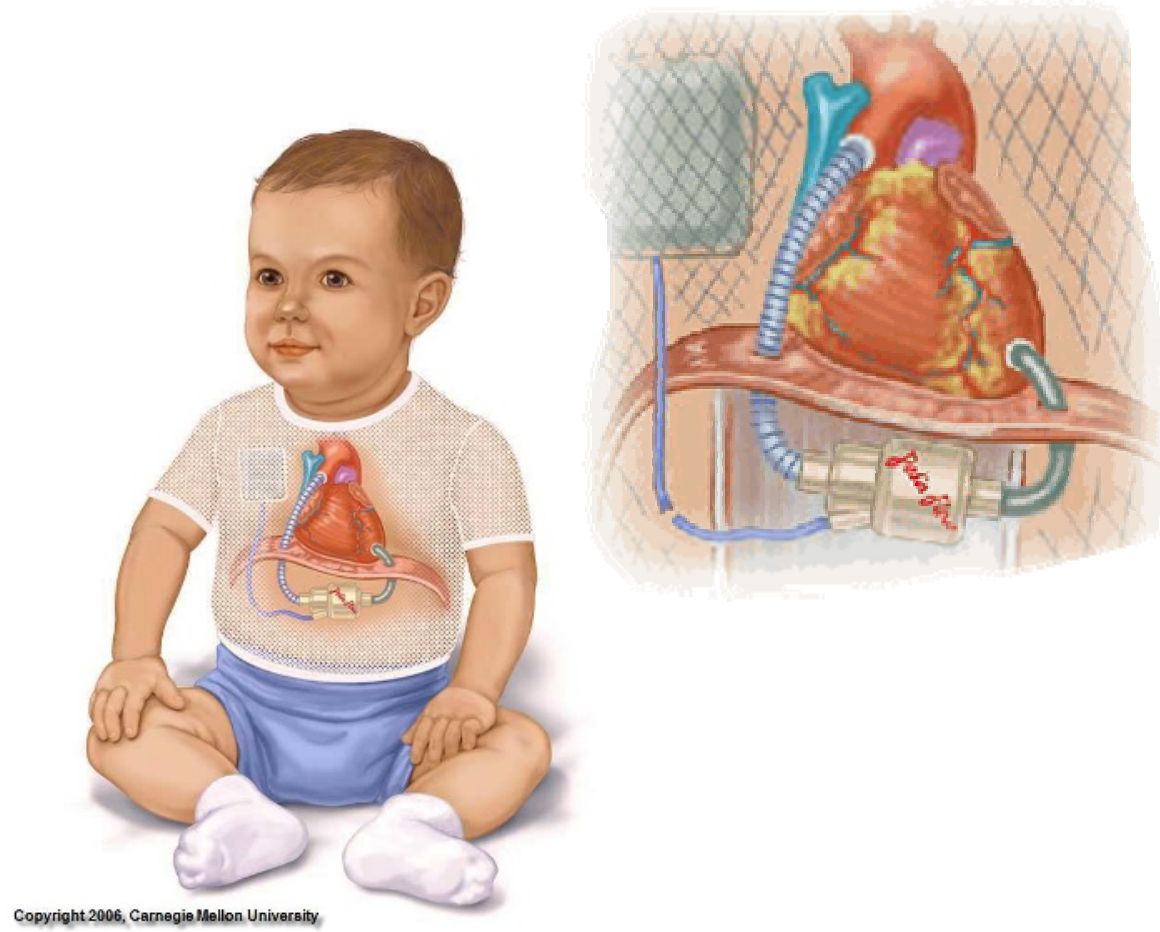

Fig. 1. Pediatric patient with an implanted pediatric ventricular assist device

Various decisions must be made concerning the placement of the VAD in addition to the decision on cannula length, diameter and angle of insertion. Advanced computer simulations are being conducted to assure compatibility with the blood; however the typical method for determining anatomic fit is usually through physical experiments on cadavers or living subjects. ${ }^{1}$ While cadaver studies allow direct anatomical dimensions to be taken; there are several disadvantages of such an approach. The major disadvantages include the limited availability of subjects and the inability to rapidly modify the implanted hardware. Additional complications due to tissue fixation, lung deflation and myocardial compression prevent accurate representation of the anatomy of living subjects. ${ }^{1}$ Intra-operative studies addresses some of these limitations but are extremely constrained in time, subject availability, and extent of manipulations that are permissible.

The purpose of this project therefore is to employ a geometric computer model to facilitate both the design and the surgical strategy to provide the optimal fit for as wide a range of patient sizes and diagnosis as possible. The anatomic data for young patients is rather sparse in the literature. Therefore an additional goal of this study is to develop a database of thoracic and abdominal measurements, similar to that developed by Mussivand et al. ${ }^{2}$ Additional advantages of 3D reconstruction include the ability to obtain anatomical measurements from live subjects, minimal invasiveness, and the ability to generate a CAD solid model manipulated for fit and cannulae design. However, there are also few challenges to using this method, which are addressed herein. 


\section{Methods}

\subsection{Acquisition of Image Data}

Retrospective images were acquired from a patient (male, 8 years of age) who underwent a high resolution gated CT scan of the chest with a 64 detector GE CT scanner (GE Medical Systems, Waukesha, WI) at the Children's Hospital of Pittsburgh. Image slice thickness was limited to $2 \mathrm{~mm}$ due to radiation dosage concerns in pediatric patients. The images were re-sliced by interpolation to $1.25 \mathrm{~mm}$ slick thickness using conventional post-processing software.

\subsection{Three-Dimensional Reconstruction}

The serial CT data were imported as DICOM files into visualization software, Mimics (Materialise, Ann Arbor, MI), to generate a 3-D surface model of the chest cavity. The raw images were manipulated by pre-processing enhancement, followed by segmentation, reconstruction and visualization, illustrated in Figure 2.

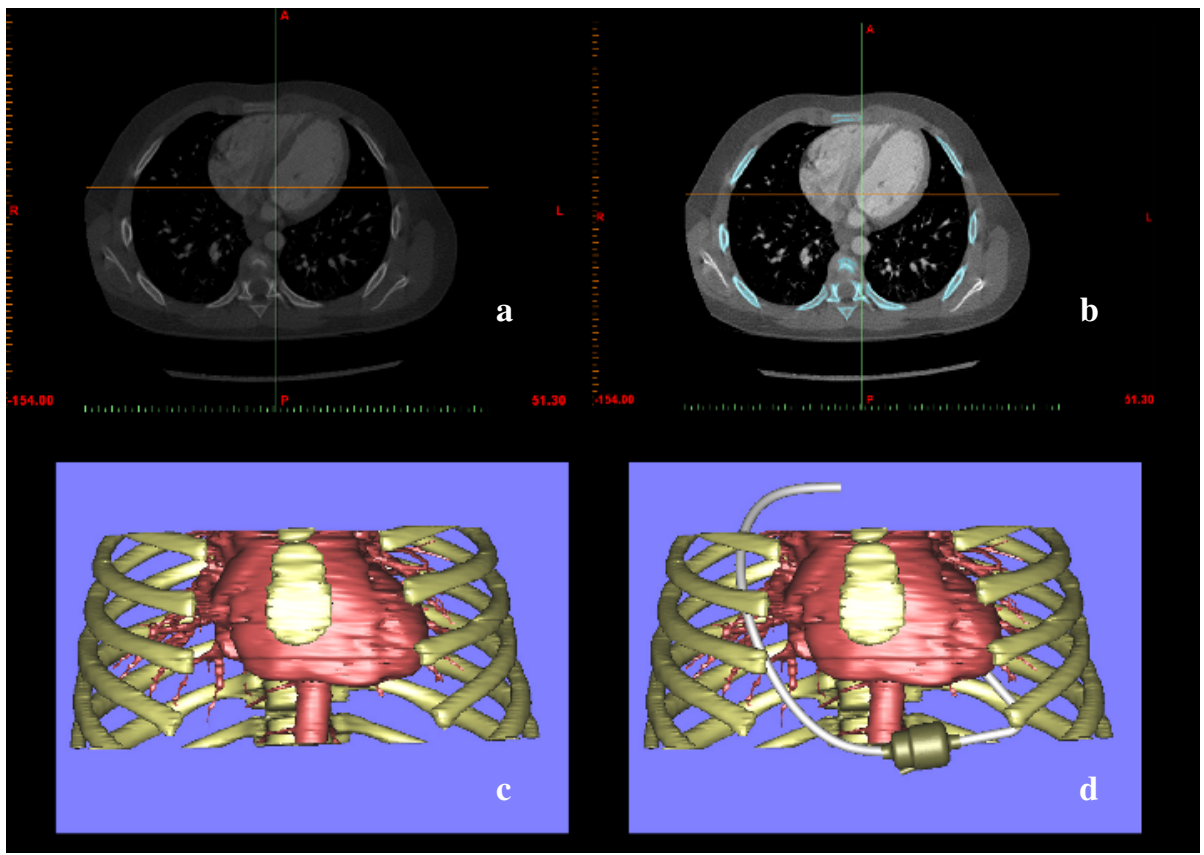

Fig. 2. 3-D reconstruction process, (a) Images are imported into Mimics and contrast-enhanced (b) Segmentation of bone by thresholding, (c) Reconstruction of ribs, heart, vessels, and bronchi (d) Introduction of solid models of implanted hardware: pediatric VAD and cannulae

\subsection{Enhancement}

Images acquired from the CT scan were enhanced to differentiate anatomical structures from each other. The DICOM files, a standard medical image file format, 
produced by the CT scanner were imported to the data visualization software, Mimics. A project file of the images was created to view the images in the software. During automatic segmentation, the surrounding structures pixels are often classified as soft tissue leading to undesirable result, therefore the images were enhanced by the manual contrast adjustment tool, through a histogram equalization method, to differentiate surrounding tissue from the heart muscle.

\subsection{Segmentation}

The cardiac boundaries were identified using a combination of methods, specifically: a thresholding tool was used to select the desired organ for reconstruction. This tool selects the organ based on the density of the pixels; generally the tool selects surrounding tissues as well. Additional editing of the boundaries was made to a number of image slices to eliminate outlier data and patch dropout regions.

\subsection{Reconstruction}

Following the segmentation of each image slice in the data series a $3 \mathrm{D}$ surface rendering was generated. Boundaries were merged to produce a continuous surface rendering. Finally, a surface contour tool was used to smooth extraneous structures from the reconstructed geometry.

\subsection{Visualization}

The final step to $3 \mathrm{D}$ reconstruction is visualization of the $3 \mathrm{D}$ image. There are various ways to manipulate the image. SolidWorks (Concord, MA), a CAD modeling software was used to visualize reconstructed anatomy. The Mimics software has the ability to export files in IGES and STL format to this modeling software. In SolidWorks, various visualization tools can be used to adjust the section view, rotation, lighting, and coloring.

\subsection{Measurements}

Anatomic measurements of pediatric patients are needed for the development of the implantable VAD in order to develop a pump that has the ability to fit in patient ranging from $2.5 \mathrm{~kg}$ to $15 \mathrm{~kg}$. Anatomic landmarks relevant to the insertion and positioning of the PVAD were recorded within Mimics. Landmarks included the position of the ribcage in reference to left ventricular apex as well as the diameter of the aorta. Using Mimics as well as SolidWorks, the described measurements were taken to prove feasibility of the measurement tool.

Additional critical measurements can be recorded using Mimics to develop a database of anthropometric data of pediatric patients in the target patient population, specifically: ${ }^{1,2}$

1. Volume ratio of the device with respect to vital organs: liver, heart, and lung.

2. Distances between organs:

a. Distance between the left ventricular apex and diaphragm.

b. Distance between the aortic arch and ventricular apex.

c. Distance between left ventricular apex and chest wall.

d. Diameter of descending aorta. 


\section{Results}

A three-dimensional model of and 8 year old male subject was constructed as shown in Figure 3. The modeled anatomy includes the chest wall (ribs, sternum and vertebrae), heart and descending aorta. Measurements of the cardiac anatomy yielded an estimated inner diameter of the aorta of $13.7 \mathrm{~mm}$. This will affect the choice of cannula used for the outlet of the VAD. The distance between the left ventricular apex to the chest was measured at $12.1 \mathrm{~mm}$. This affects the ability to access the heart by the inflow cannula.

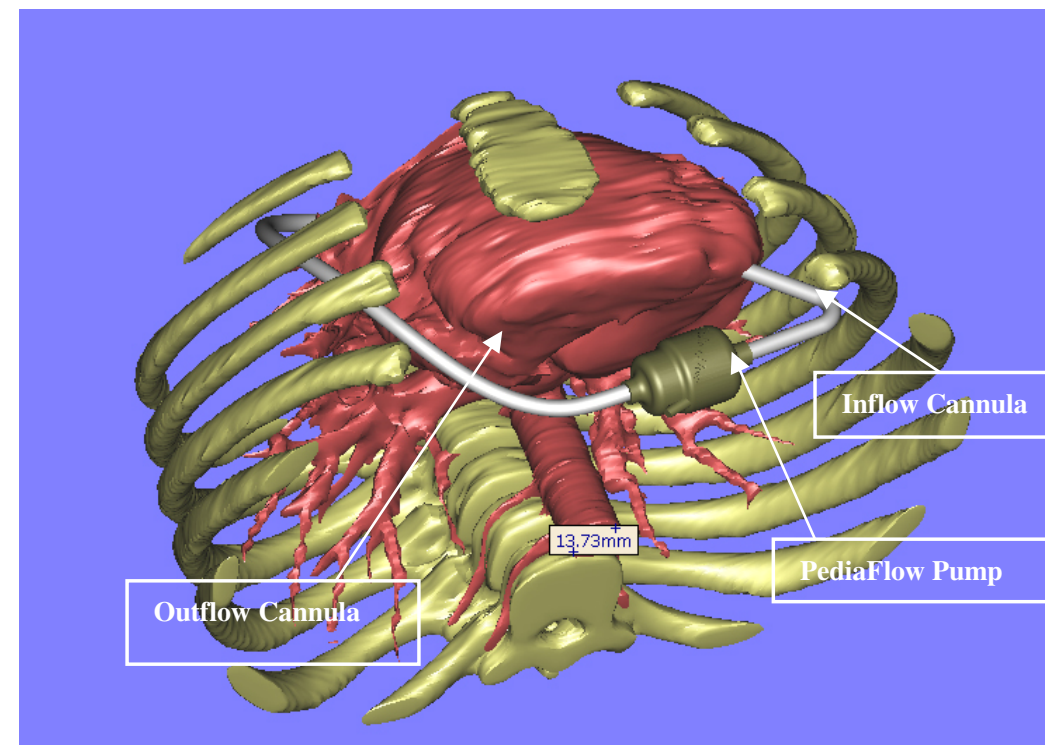

Fig. 3. 3-D reconstruction of the chest, heart, PVAD (gold) and cannula (white). Measurement of internal diameter of the descending aorta was $13.7 \mathrm{~mm}$.

Various locations were considered for locating the implanted pump. The $\mathrm{x}, \mathrm{y}, \mathrm{z}$ locations as well as the "patch", "yaw", \& "roll" angles were varied to achieve quantitatively and compromise between:

- Length of cannula, hence pressure drop (minimize)

- Compression/ abrasion of organs (minimize)

- Target sites conveniently assessable.

- Kinks or sharp bends that might obstruct or disturb flow.

Figure 3 depicts one preferred configuration with the pump positioned below the diaphragmatic margin. Due to the relative size of the current VAD with respect to chest cavity, the preferred location of the VAD will be in the abdomen with cannula penetrating the diaphragm. Hence both abdominal and thoracic surgery will be required. 
Cannulae constructed in SolidWorks were modified to connect the VAD from the LV apex to the ascending aorta. The outflow cannula was designed to be $70 \mathrm{~mm}$ in length and diameter of $5 \mathrm{~mm}$.

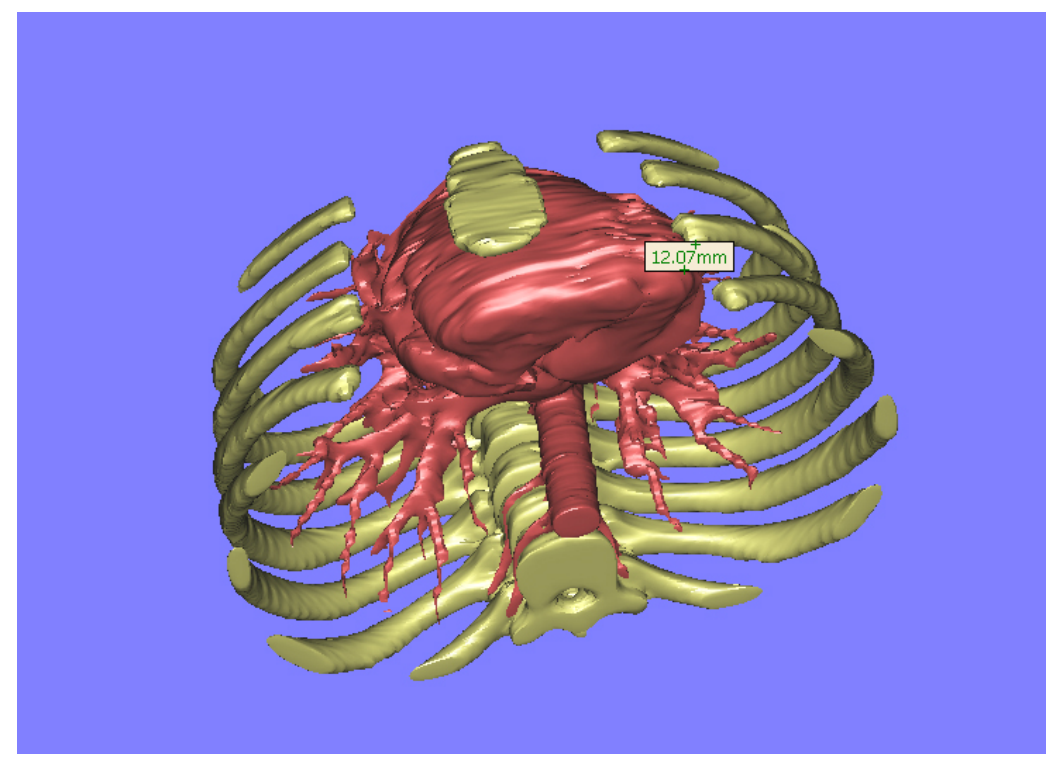

Fig. 4. Anterior measurement of distance between the left ventricular apex and the ribcage

\section{Discussion}

Using Mimics and SolidWorks, it was possible to generate a three-dimensional solid assembly to simulate the implantation of a Pediatric VAD in the chest of a young patient. These models can be easily measured and manipulated for the anatomical fit studies as described by Warriner et al. ${ }^{5}$ The models have proven helpful for development of the cannulae, specifically to determine the proper insertion angle into the LV chamber and anastomosis location at the aorta. ${ }^{6}$ These results will enable future computational fluid dynamic simulations to optimize the geometry according to flow requirements.

However there are still several challenges to pursuit of $3 \mathrm{D}$ reconstruction of pediatric patients. Foremost, the spatial resolution required for accurate representation necessitates CT data series with very fine slice thickness, ideally less than $1 \mathrm{~mm}$. Additional cardiac gating is needed to obtain temporal accuracy and avoid geometric distortion throughout the cardiac cycle. Obtaining these data however would require radiation dosage exceeding levels that would typically be indicated.

Secondly, segmentation, a perennial problem, is exacerbated in these very small patients due to limited resolution and contrast. Manually editing is almost always necessary, and it is unlikely that the process will be fully automated. 
Future work will entail collection of additional data as well as utilization of the current data set for subsequent simulation studies. Future image collection will include a prospective study of brain dead patients which will enable slice thickness down to $0.63 \mathrm{~mm}$. By collecting data over a statistical sampling of patients, it will be possible to develop an anthropometric database of thoracic and abdominal anatomy that may be made available for a variety of purposes. Validation of accuracy for the generation of 3D models by a human expert will be accomplished by obtaining cadaveric measurements shortly after the brain dead patients are withdrawn from ventilator support. The cadaveric measurements will be compared to the measurements acquired from the 3D models. Ongoing research with the current data set includes flow visualization experiments based on transparent replicas of the fluid geometry. Combined with computational fluid dynamics simulation, these studies will collectively be used to optimize the biocompatibility efficacy, and overall safety of the pediatric ventricular assist device.

\section{References}

1. Zhang, B.M., T. Tatsumi, E. Taenaka, Y. Uyama, C. Takano, H. Takamiya, M., ThreeDimensional Thoracic Modeling for an Anatomical Compatibility Study of Implantable Total Artificial Heart. Artificial Heart, 1999. 23(3): p. 229-234.

2 Mussivand, T., et al., Critical anatomic dimensions for intrathoracic circulatory assist devices. Artif Organs, 1992. 16(3): p. 281-5.

3. Fujimoto, L.K., et al., Human thoracic anatomy based on computed tomography for development of a totally implantable left ventricular assist system. Artif Organs, 1984. 8(4): p. 436-44.

4. Fujimoto, L.K., et al., Anatomical considerations in the design of a long-term implantable human left ventricle assist system. Artif Organs, 1985. 9(4): p. 361-74.

5. Warriner, R.K., et al., Virtual anatomical three-dimensional fit trial for intra-thoracically implanted medical devices. Asaio J, 2004. 50(4): p. 354-9.

6. May-Newman, K.D., Hillen, B.K, Sironda, C. S, Dembitsky, W., Effect of LVAD outflow conduit insertion angle on flow through the native aorta. Journal of Medical Engineering \& Technology, 2004. 28(3): p. 105-109. 